\title{
Non-immunologic mechanisms of calcineurin inhibitors explain its antiproteinuric effects in genetic glomerulopathies
}

\author{
Albert Bensman • Patrick Niaudet \\ Received: 30 November 2009 / Accepted: 30 November 2009 / Published online: 2 March 2010 \\ (C) IPNA 2010
}

\begin{abstract}
It has been reported (this issue Pediatric Nephrology) that cyclosporine A (CyA) therapy in combination with corticosteroids, angiotensin-converting enzyme inhibitor, and an angiotensin receptor blocker decreased proteinuria in three patients with nephrotic syndrome (NS) due to WT1 mutations. Treatment with calcineurin inhibitors were found to induce a partial remission of proteinuria in several other children with genetic forms of NS, such as mutation in the podocine and in the phospholipase $\mathrm{C}$ epsilon gene. CyA therapy has also been reported to be beneficial to patients with Alport syndrome. Recent data have shown that the antiproteinuric effect of CyA in these cases may be due to a non-immunologic mechanism. CyA exerts an antiproteinuria effect by preventing the degradation of the actin organizing protein synaptodpodin and by a downregulation of TRPC6. This mechanism leads to the stabilization of the actin cytoskeleton in the kidney podocytes. This beneficial effect of CyA is interesting, but long-term results regarding function and nephrotoxicity are still missing.
\end{abstract}

Keywords Actin cytoskeleton - Calcineurin inhibitors . Genetic nephrotic syndrome · Podocytes · Synaptopodin

A. Bensman $(\bowtie)$

Service de néphrologie pédiatrique,

Hôpital d'enfants Armand Trousseau,

26 avenue du Dr A. Netter,

75572 Paris, Cédex 12, France

e-mail: a.bensman@trs.aphp.fr

P. Niaudet

Service de néphrologie pédiatrique, Hôpital des Enfants Malades,

Paris, France

\section{Introduction}

In this issue of Pediatric Nephrology, Gellermann et al. [1] report that patients with $W T 1$ mutations may respond to cyclosporine A (CyA) therapy given in combination with corticosteroids. In their study, CyA had a beneficial effect on proteinuria in three cases of nephrotic syndrome (NS) due to WT1 mutations. It is also important to note, however, that the addition of an angiotensin-converting enzyme (ACE) inhibitor and an angiotensin receptor blocker to the therapeutic regimen may have played an additional role in the reduction of proteinuria in these patients.

The treatment of glomerular diseases with CyA was first described more than 20 years ago [2]. CyA has been found to induce the remission of proteinuria in patients with minimal change disease in which a $\mathrm{T}$ cell dysfunction is thought to play a crucial role. It has been postulated that CyA acts through the inhibition of NFAT signalling in $T$ lymphocytes [3], but as the immune system is not involved in the glomerular disease due to WT1 mutations, the effect of CyA must therefore be related to other mechanisms [4]. However, long-term results on renal function and nephrotoxicity in this context are not yet available.

\section{CyA in other non-immunological forms of NS}

Malina et al. reported a partial remission of proteinuria in a child with a NS due to a NPHS2 mutation following treatment with CyA. The CyA therapy had no negative effects on renal function [5].

Mutations in the phospholipase $\mathrm{C}$ epsilon gene may cause early-onset NS with end-stage kidney disease (ESRD) [6]. Histological examination of kidney tissue obtained from affected individuals has revealed either 
diffuse mesangial sclerosis or focal segmental glomerulosclerosis. Notably, Hinkes et al. described two children with truncating mutations of the phospholipase $\mathrm{C}$ epsilon gene who responded to treatment with corticosteroids or CyA [6]. One child responded to an initial 4-month course of CyA treatment, which was subsequently extended to 2.5 years.

CyA has also been reported to reduce proteinuria in patients with Alport syndrome. Callis et al. reported on eight male patients with Alport syndrome treated with CyA for 7-10 years. These patients showed a slower progression to renal failure than their affected but untreated siblings $[7,8]$. Repeat renal biopsies did not show any signs of CyA nephrotoxicity after 5 years of treatment. In another study, Charbit et al. found that the same doses of CyA resulted in a significant reduction of proteinuria as well as an increase in serum albumin in eight of nine patients [9]. However, CyA was rapidly responsible for nephrotoxicity in seven patients who experienced a $18-52 \%$ reduction of inulin clearance with 6 months of treatment. Serial renal biopsies performed on five patients who had continued the treatment for 20-27 months revealed that four of these showed marked worsening of renal lesions.

Chen et al. studied the effects of CyA in a canine model of X-linked Alport syndrome [10] and found that CyA was beneficial in slowing the progression of the disease in terms of glomerular filtration rate (GFR) and pathological lesions.

\section{Mechanisms of the beneficial effect of CyA}

Mechanisms other than the immunosuppressive effects of CyA are involved in the reduction of proteinuria in patients with glomerular diseases since CyA significantly decreases proteinuria in a number of glomerulopathies with no immunological background [3].

One proposed mechanism is the afferent arteriole vasoconstriction induced by CyA. The amount of serum albumin crossing the glomerular filtration barrier is reduced due to the reduction in the glomerular filtration rate. Zietse et al. studied the effects of CyA on renal perfusion and the glomerular filtration barrier in patients with NS of various etiology [11]. Low doses of CyA led to the restoration of the charge selectivity of the glomerular barrier in minimal change disease, whereas in membranous glomerulopathy, changes in proteinuria were related to an increase in glomerular permselectivity and a decrease in filtrate flow through the shunt pathway. Proteinuria did not decrease in patients with membranoproliferative glomerulonephritis or focal and segmental glomerulosclerosis despite the hemodynamic effects of CyA.

In an experimental model of anti-glomerular capillary basement membrane (GBM) nephritis, Schrijver et al. showed that CyA had an antiproteinuric effect independent from the glomerular hemodynamic changes [12]. They postulated a reduction of the permselectivity of the glomerular barrier for proteins. This hypothesis is in accordance with the findings of Ambalavanan et al. [13]. These researchers studied 41 patients with NS and biopsyproven membranous nephropathy who received a 3- to 6-month course of CyA. They found that the GFR and renal plasma flow remained constant but that the CyA restored the destran-sieving curve towards normal, thereby lowering the computed fraction of shunt-like pores by $25 \%$. Interestingly, Charbit et al. found the opposite results in patients with Alport syndrome [9]. These researchers performed fractional neutral dextran clearances in nine children treated with CyA and observed no difference in the size selectivity for molecules with a radius of $30-54 \AA$ prior to and following CyA therapy.

Chen et al. proposed that the beneficial effect of CyA in their canine model came from a delayed deterioration of the GBM structure, which in turn could be related to glomerular haemodynamics altered by CyA [10].

In two individuals with PLCE1 truncating mutations who showed full and sustained treatment responses, Hinkes et al. [6] speculated that there may be a critical time window in glomerular development during which treatment with glucocorticoids or CyA may overcome a putative developmental defect imposed by the PLCE1 loss of function. This may occur through the induction of a redundant mechanism, such as the activity of another phospholipase $\mathrm{C}$. This concept that the arrest of glomerular development through PLCE1 mutations may be reversible by treatment with glucocorticoids or CyA is very interesting. The exact mechanism has yet to be elucidated.

Faul et al. proposed yet another mechanism for the antiproteinuric effect of CyA [14]. It is known that the filter function of a normal kidney requires a normal podocyte actin cytoskeleton. Synaptopodin is an actin binding protein that is highly expressed in podocytes and which is an important regulator of podocyte function through its interaction with CD2AP. Indeed, bigenic heterozygosity for both proteins causes proteinuria and focal segmental glomerulosclerosis (FSGS). Faul et al. showed that the activation of calcineurin in the podocytes results in the dephosphorylation of synaptopodin and causes proteinuria via the degradation of synaptopodin. These results suggest that the antiproteinuric effect of CyA is the result of a direct effect on synaptopodin via the maintenance of synaptopodin abundance in podocytes [15]. This mechanism leads to the stabilization of its actin cytoskeleton.

TRPC6, a transient receptor potential channel, increases calcium influx in the podocytes. Gain-of-function mutations of the TRPC6 gene cause familial FSGS, and TRPC6 overexpression have been found in acquired proteinuria. 
Nijenhuis et al. found TRPC6 overexpression in the animal model of adriamycin nephropathy that correlated with glomerular damage [16]. Angiotensin receptor blockade or ACE inhibitors ameliorate the upregulation of TRPC6. These authors also showed that angiotensin II and adriamycin stimulate TRPC6 expression in cultured podocytes through the calcineurin pathway. Therefore, CyA, through calcineurin inhibition, downregulates TRPC6 expression in vitro and in vivo in adriamycin nephropathy, resulting in reduced proteinuria. Interestingly, TRPC6 gene deficiency is associated with less proteinuria in adriamycin nephropathy [17].

\section{Conclusion}

Calcineurin inhibitors decrease proteinuria through different mechanisms, with some of these mechanisms being unrelated to the inhibition of the NFAT signaling in $\mathrm{T}$ lymphocytes. Recent findings showing that CyA exerts an antiproteinuric effect by preventing the degradation of synaptopodin and by a downregulation of TRPC6 expression open new avenues for the search of new drugs without the nephrotoxic effects of calcineurin inhibitors.

The beneficial effect of CyA in the three cases of nephrotic syndrome due to WT1 mutation is interesting, but it does not indicate that CyA therapy slows the progression of the disease to ESRD. Moreover, the potential nephrotoxicity of the drug may limit its long-term use.

\section{References}

1. Gellermann J, Stefanidis CJ, Mitsioni A, Querfeld U (2010) Successful treatment of steroid-resistant nephrotic syndrome associated with WT1 mutations. Pediatr Nephrol. doi:10.1007/s00467-010-1468-3

2. Meyrier A (1989) Treatment of glomerular disease with cyclosporin A. Nephrol Dial Transplant 4:923-931

3. Meyrier A (2009) Treatment of focal segmental glomerulosclerosis with immunophilin modulation: when did we stop thinking about pathogenesis? Kidney Int 76:487-491

4. Meyrier A (1992) Antiproteinuric and immunological effects of cyclosporin $\mathrm{A}$ in the treatment of glomerular diseases. Nephrol Dial Transplant 7[Suppl 1]:80-84
5. Malina M, Cinek O, Janda J, Seeman T (2009) Partial remission with cyclosporine A in a patient with nephrotic syndrome due to NPHS2 mutation. Pediatr Nephrol 24:2051-2053

6. Hinkes B, Wiggins RC, Gbadegesin R, Vlangos CN, Seelow D, Nurnberg G, Garg P, Verma R, Chaib H, Hoskins BE, Ashraf S, Becker C, Hennies HC, Goyal M, Wharram BL, Schachter AD, Mudumana S, Drummond I, Kerjaschki D, Waldherr R, Dietrich A, Ozaltin F, Bakkaloglu A, Cleper R, Basel-Vanagaite L, Pohl M, Griebel M, Tsygin AN, Soylu A, Muller D, Sorli CS, Bunney TD, Katan M, Liu J, Attanasio M, O’Toole JF, Hasselbacher K, Mucha B, Otto EA, Airik R, Kispert A, Kelley GG, Smrcka AV, Gudermann T, Holzman LB, Nurnberg P, Hildebrandt F (2006) Positional cloning uncovers mutations in PLCE1 responsible for a nephrotic syndrome variant that may be reversible. Nat Genet 38:1397-1405

7. Callis L, Vila A, Carrera M, Nieto J (1999) Long-term effects of cyclosporine A in Alport's syndrome. Kidney Int 55:1051-1056

8. Callis L, Vila A, Nieto J, Fortuny G (1992) Effect of cyclosporin A on proteinuria in patients with Alport's syndrome. Pediatr Nephrol 6:140-144

9. Charbit M, Gubler MC, Dechaux M, Gagnadoux MF, Grunfeld JP, Niaudet P (2007) Cyclosporin therapy in patients with Alport syndrome. Pediatr Nephrol 22:57-63

10. Chen D, Jefferson B, Harvey SJ, Zheng K, Gartley CJ, Jacobs RM, Thorner PS (2003) Cyclosporine A slows the progressive renal disease of alport syndrome (X-linked hereditary nephritis): results from a canine model. J Am Soc Nephrol 14:690-698

11. Zietse R, Wenting GJ, Kramer P, Schalekamp MA, Weimar W (1992) Effects of cyclosporin A on glomerular barrier function in the nephrotic syndrome. Clin Sci 82:641-650

12. Schrijver G, Assmann KJ, Wetzels JF, Berden JH (1995) Cyclosporin A reduces albuminuria in experimental anti-GBM nephritis independently from changes in GFR. Nephrol Dial Transplant 10:1149-1154

13. Ambalavanan S, Fauvel JP, Sibley RK, Myers BD (1996) Mechanism of the antiproteinuric effect of cyclosporine in membranous nephropathy. J Am Soc Nephrol 7:290-298

14. Faul C, Donnelly M, Merscher-Gomez S, Chang YH, Franz S, Delfgaauw J, Chang JM, Choi HY, Campbell KN, Kim K, Reiser J, Mundel P (2008) The actin cytoskeleton of kidney podocytes is a direct target of the antiproteinuric effect of cyclosporine A. Nat Med 14:931-938

15. Asanuma K, Kim K, Oh J, Giardino L, Chabanis S, Faul C, Reiser J, Mundel P (2005) Synaptopodin regulates the actin-bundling activity of alpha-actinin in an isoform-specific manner. J Clin Invest 115:1188-1198

16. Nijenhuis T, Hoenderop J, Flesche J, van Goor H, Bakker M, Bindels R, Navis G, Wetzels J, Berden J, Reiser J, van der Viag J (2009) Angiotensin II-mediated upregulation of TRPC6 expression via calcineurin/NFAT signaling in podocyte injury (abstract TH-P0910). J Am Soc Nephrol 20:319A

17. Gbadegesin RA, Domadaran TV, Homstad A, Bartkowiak B, Bowling B, Wy G, Lavin P, Eckel J, Mukerji N, Winn M (2009) TRPC6 gene deficiency ameliorates the course of puromycin induced kidney injury (abstract TH-PO907). J Am Soc Nephrol 20:318A 